\title{
Mothership versus Drip-and-Ship Model for Mechanical Thrombectomy in Acute Stroke: A Systematic Review and Meta-Analysis for Clinical and Radiological Outcomes
}

\author{
Michele Romoli, ${ }^{\mathrm{a}, \mathrm{b}}$ Maurizio Paciaroni, ${ }^{\mathrm{c}}$ Georgios Tsivgoulis, ${ }^{\mathrm{d}, \mathrm{e}}$ Elio Clemente Agostoni, ${ }^{\mathrm{f}}$ \\ Simone Vidale ${ }^{\mathrm{a}}$ \\ aNeurology Unit, Rimini "Infermi" Hospital, AUSL Romagna, Rimini, Italy \\ ${ }^{b}$ Neurology Clinic, Santa Maria della Misericordia Hospital, University of Perugia, Perugia, Italy \\ 'Stroke Unit and Division of Cardiovascular Medicine, Santa Maria della Misericordia Hospital, University of Perugia, Perugia, Italy \\ 'Second Department of Neurology, "Attikon" Hospital School of Medicine, National and Kapodistrian University of Athens, Athens, Greece \\ eDepartment of Neurology, University of Tennessee Health Science Center, Memphis, TN, USA \\ fDepartment of Neurology, Niguarda Ca' Granda Hospital, Milan, Italy
}

Background and Purpose Substantial uncertainty exists on the benefit of organizational paradigms in stroke networks. Here we systematically reviewed and meta-analyzed data from studies comparing functional outcome between the mothership (MS) and the drip and ship (DS) models. Methods The meta-analysis protocol was registered international prospective register of systematic reviews (PROSPERO) and followed Preferred Reporting Items for Systematic Reviews and MetaAnalyses (PRISMA) guidelines. PubMed, EMBASE, and Cochrane Central databases were searched for randomized-controlled clinical trials (RCTs), retrospective and prospective studies comparing MS versus DS. Primary endpoints were functional independence at 90 days (modified Rankin Scale $<3$ ) and successful recanalization (Thrombolysis in Cerebral Infarction Scale $[\mathrm{TICl}]>2 \mathrm{a}$ ); secondary endpoints were 3-month mortality and symptomatic intracranial haemorrhage ( $\mathrm{s} \mathrm{CH})$. Odds ratios for endpoints were pooled using the random effects model and were compared between the two organizational models.

Results Overall, 18 studies $(n=7,017)$ were included in quantitative synthesis. MS paradigm was superior to DS model for functional independence (odds ratio, 1.34; 95\% confidence interval, 1.16 to $1.55 ; I^{2}=30 \%$ ). Meta-regression analysis revealed association between onset-to-needle time and good functional outcome, with longer onset-to-needle time being detrimental. Similar rates of recanalization, sICH and mortality at 90 days were documented between MS and DS.

Conclusions Patients with acute ischemic stroke eligible for reperfusion strategies might benefit more from MS paradigm as compared to DS. RCTs are needed to further refine best management taking into account logistics, facilities and resources.
Correspondence: Simone Vidale Department of Neurology, Infermi Hospital, Viale Luigi Settembrini 2, 47923 Rimini, Italy

Tel: +39-0541705600 E-mail: simone.vidale@ausIromagna.it https://orcid.org/0000-0003-14260885

Received: May 14, 2020

Revised: August 7, 2020

Accepted: August 25, 2020

Keywords Stroke; Mothership; Drip and ship; Thrombectomy; Endovascular procedures 


\section{Introduction}

Previous randomized-controlled clinical trials (RCTs) showed that the combination of intravenous thrombolysis (IVT) and endovascular thrombectomy (EVT) is effective in patients with ischemic stroke due to a large vessel occlusion. ${ }^{1-3}$ Current international guidelines have been updated accordingly. ${ }^{4}$ However, EVT is only available at Comprehensive Stroke Centers (CSC), which are fewer than Primary Stroke Centers (PSC), the latter being only able to administer IVT. Therefore, two main organizational paradigms have been developed: the mothership (MS), in which the patient is directly brought to the $\mathrm{CSC}$, and the drip and ship (DS) model, where initially assessment and eventual IVT at the PSC are followed by "shipping" to the CSC. The choice of a model over another implies clinical consequences for treated patients as well as for local health policies, including the distribution of hospital facilities over the region of interest.

Previous systematic reviews comparing MS versus DS models were limited to few studies and provided conflicting results., ${ }^{5,6}$ Computational modeling provided potential insights on time of transport, although with consistent limitations due to assumptions of treatment efficacy and patient eligibility. Therefore, no conclusive evidence is available to date concerning the superiority of one model over the other by clinical and economic results.

We conducted a systematic review and pooled data metaanalysis of studies comparing MS versus DS model, including subgroup analysis by type of treatment, clinical severity and timing of treatments.

\section{Methods}

\section{Search strategy}

The methods and guidelines of this study-level meta-analysis followed Preferred Reporting Items for Systematic Reviews and Meta-Analyses (PRISMA) guidelines ${ }^{8}$ and study protocol registered with PROSPERO (CRD42019135915). Two reviewers systematically searched Pubmed, EMBASE and Cochrane Central register of Controlled Trials databases for studies comparing MS versus DS published between January 1990 and February 1st, 2020. Search strategy was based on combination of terms, including "mothership," "drip and ship," "organization model," "stroke," "thrombolysis," "thrombectomy," as either keywords or $\mathrm{MeSH}$ terms. Reference lists and citing articles were also reviewed to increase the identification of relevant studies.

\section{Selection criteria}

We included RCTs, prospective and retrospective studies re- porting the clinical efficacy and safety of MS or DS model among adult $(\geq 18)$ patients with acute ischemic stroke, independently from the device used. We limited the studies to English language and excluded case reports, small case series $(<20)$, conference proceedings and reviews. The interventional group comprised patients treated in a MS model, while the control group was represented by the DS paradigm.

\section{Endpoints}

The primary endpoint was functional independence at 90 days from stroke onset, defined as modified Rankin Scale score $<3$. Secondary endpoints were (1) rate of good recanalization according to Thrombolysis in Cerebral Infarction Scale ( $\mathrm{TICl}$ ) grade (2b or 3), (2) mortality at 90 days from stroke onset, (3) and the occurrence of symptomatic intracerebral haemorrhage (sICH), according to the definition of individual studies.

\section{Data extraction and bias assessment}

Two reviewers independently extracted data concerning baseline and outcome characteristics of each included study, as well as its methodological design. We reported the lack of data on outcome, when appropriate. Risk of bias was assessed and reported according to the recommendations of the Cochrane Handbook for Systematic Reviews of Intervention, applying the Cochrane risk of bias tool or the Newcastle-Ottawa Scale for bias assessment when appropriate. Funnel plots were implemented for publication bias.

\section{Statistical analysis}

We performed a statistical analysis pooling data in the intervention group and the control group. Outcome heterogeneity was evaluated with Cochrane's 0 test $I^{2}$. We calculated odds ratio (ORs) and 95\% confidence intervals (Cls), with a random effects model, for all outcomes. We calculated $\mathrm{I}^{2}$ statistics, and heterogeneity was classified as moderate $\left(1^{2} 30 \%\right.$ to $\left.50 \%\right)$, substantial (I $\left.\right|^{2} 50 \%$ to $\left.75 \%\right)$, or considerable $\left(I^{2} 75 \%\right.$ to $\left.100 \%\right)$. If the results were heterogenous, we planned to use sensitivity analysis to investigate how the results differed when we excluded studies with highest risk of bias. Subgroup analysis for the primary outcome was predefined for different types of treatment, including studies considering bridging therapy only. Sensitivity analysis through leave-one out paradigm was used to test robustness of the findings. We report the analysis results graphically using forest plots for outcomes of single included trials and the total treatment effects. We also calculated the number needed to treat (NNT) for the primary endpoint using the formula NNT=1/[(1-RR) $\times$ mortality rate in control group]. Finally, we introduced covariates to reduce heterogene- 


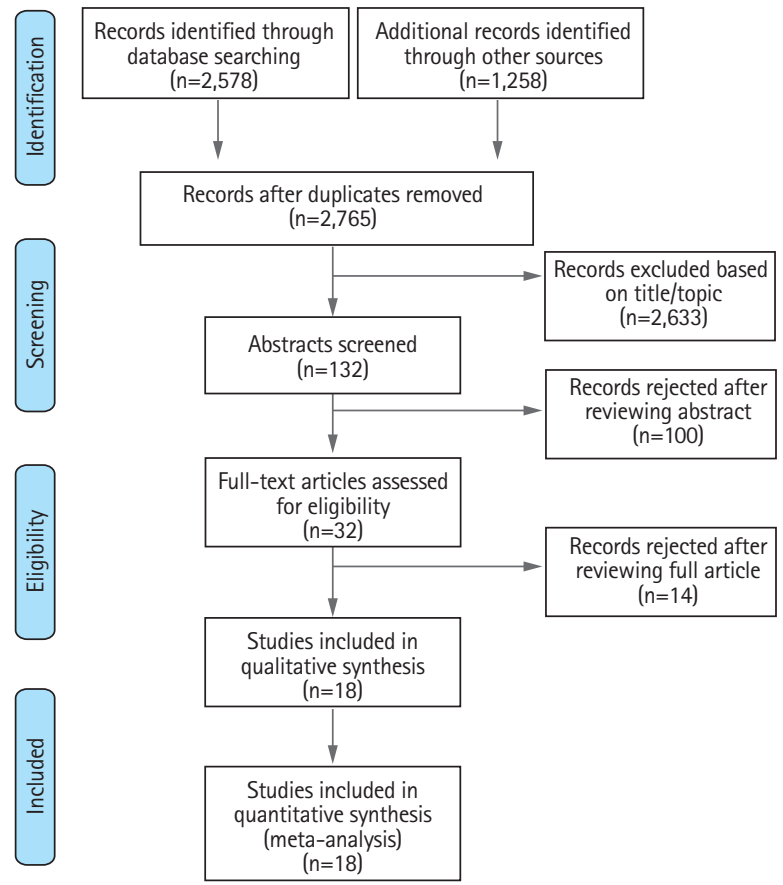

Figure 1. Preferred Reporting Items for Systematic Reviews and MetaAnalyses (PRISMA) flow-chart for selection of studies included in the metaanalysis. ity of meta-analysis and we performed a meta-regression analysis to detect the influence of these variables on the primary endpoint. In particular, we considered the differences of means between interventional and control groups of each included study concerning age, clinical severity summarized by the score of National Institutes of Health Stroke Scale (NIHSS), onset-to-groin time (OGT) and onset-to-needle time (ONT). We reported graphical representation of final results by bubbleplots. Data analysis was performed using Review Manager version 5.3 (The Cochrane Collaboration 2012, Copenhagen, Denmark) and R software version 3.3.1 (packages metaphor, ${ }^{9}$ meta; R Foundation for Statistical Computing, Vienna, Austria).

\section{Results}

Systematic search retrieved 18 studies including 7,017 patients (PRISMA flowchart) (Figure 1). ${ }^{10-27}$ Most of the included studies were observational and prospective, only one study was a $\mathrm{RCT}^{15}$ (Table 1). As a result, quality of studies ranged fair to good, with only one RCT achieving optimal score on risk of bias assessment. Funnel plot showed low levels of visual asymmetry (Supplementary Figure 1 for complete bias assessment). No significant differences emerged comparing patients treated in

Table 1. Summary of included studies

\begin{tabular}{|c|c|c|c|c|c|c|c|c|c|c|c|}
\hline \multirow{2}{*}{ Study } & \multirow{2}{*}{$\begin{array}{l}\text { Age } \\
\text { (yr) }\end{array}$} & \multicolumn{3}{|c|}{ Sample } & \multirow{2}{*}{ NIHSS } & \multicolumn{2}{|c|}{ ONT (min) } & \multicolumn{2}{|c|}{ OGT (min) } & \multicolumn{2}{|c|}{ No. of IVT (\%) } \\
\hline & & Total & Mothership & Drip \& Ship & & Mothership & Drip \& Ship & Mothership & Drip \& Ship & Mothership & Drip \& Ship \\
\hline Adams et al. (2019) ${ }^{10}$ & 72 & 214 & 124 & 90 & 16 & NA & NA & 131 & 248 & $52(42)$ & $49(54)$ \\
\hline $\begin{array}{l}\text { Cappelen-Smith et al. } \\
(2016)^{19}\end{array}$ & 67 & 33 & 20 & 13 & 21 & NA & NA & NA & NA & $7(35)$ & $9(69)$ \\
\hline Feil et al. (2020) ${ }^{11}$ & 71 & 410 & 221 & 189 & 16 & 95 & 95 & 152 & 256 & 255 (overall) & \\
\hline Froehler et al. (2017) ${ }^{17}$ & 68 & 906 & 498 & 408 & 17 & 89 & 98 & 137 & 237 & $329(66)$ & $299(73)$ \\
\hline Gerschenfeld et al. (2017) & 72 & 159 & 59 & 100 & 16 & 135 & 150 & 189 & 248 & $59(100)$ & $100(100)$ \\
\hline Hiyama et al. (2016) 22 & 75 & 45 & 12 & 33 & 20 & NA & NA & 166 & 189 & $12(100)$ & $33(100)$ \\
\hline Jayaraman et al. (2020) ${ }^{12}$ & 76 & 232 & 88 & 144 & 18 & $50^{*}$ & $62^{*}$ & 93 & 152 & $48(55)$ & $94(65)$ \\
\hline Kim et al. (2016) $)^{18}$ & 67 & 820 & 678 & 142 & 9 & 110 & 161 & NA & NA & $678(100)$ & $142(100)$ \\
\hline Mourand et al. (2019) ${ }^{14}$ & 69 & 179 & 93 & 86 & 18 & 165 & 152 & 215 & 315 & $48(52)$ & $70(81)$ \\
\hline Park et al. $(2016)^{23}$ & 69 & 105 & 77 & 28 & 12 & NA & NA & 219 & 300 & $77(100)$ & $28(100)$ \\
\hline Park et al. (2016) $)^{24}$ & 68 & 1,898 & 1,599 & 299 & 11 & $113^{+}$ & $120^{+}$ & 200 & 305 & $1,599(100)$ & $299(100)$ \\
\hline Pfaff et al. $(2017)^{16}$ & 65 & 112 & 74 & 38 & 19 & NA & NA & 178 & 283 & $54(73)$ & $29(76)$ \\
\hline Prothmann et al. $(2017)^{26}$ & 68 & 87 & 37 & 50 & 15 & NA & NA & 137 & 233 & $23(62)$ & $35(70)$ \\
\hline Rinaldo et al. $(2017)^{25}$ & 66 & 140 & 62 & 78 & 18 & NA & NA & 277 & 420 & $40(65)$ & $42(54)$ \\
\hline Saver et al. (2015) & 65 & 98 & 67 & 31 & NA & NA & NA & 275 & 180 & NA & NA \\
\hline $\begin{array}{l}\text { van Veenendaal et al. } \\
(2018)^{27}\end{array}$ & 70 & 178 & 50 & 128 & 17 & NA & NA & 158 & 293 & $32(64)$ & $97(76)$ \\
\hline Weber et al. $(2016)^{20}$ & 71 & 643 & 300 & 343 & 15 & $92^{+}$ & $115^{\ddagger}$ & 150 & 233 & NA & NA \\
\hline $\begin{array}{l}\text { Weisenburger-Lile et al. } \\
(2019)^{13}\end{array}$ & 67 & 971 & 298 & 673 & 16 & 131 & 150 & 171 & 260 & $298(100)$ & $673(100)$ \\
\hline
\end{tabular}

NIHSS, National Institutes of Health Stroke Scale; ONT, onset-to-needle time; OGT, onset-to-groin time; IVT, intravenous thrombolysis; NA, not available. ${ }^{*}$ Onset time replaced by scene departure; ${ }^{+}$Includes patients treated with bridging; ${ }^{\dagger}$ Data for whole cohort, including patients lost to follow-up. 
Table 2. Differences between groups

\begin{tabular}{lccc}
\hline Variable & $\begin{array}{c}\text { Mothership } \\
(\mathrm{n}=4,338)\end{array}$ & $\begin{array}{c}\text { Drip \& Ship } \\
(\mathrm{n}=2,808)\end{array}$ & $P$ \\
\hline Male sex (\%) & 56 & 55 & 0.406 \\
Age $(\mathrm{yr})$ & $69.6 \pm 11.0$ & $69.1 \pm 11.0$ & 0.065 \\
NIHSS score at admission & $15.7 \pm 5.0$ & $15.6 \pm 5.0$ & 0.409 \\
ONT (min) & $120 \pm 27$ & $132 \pm 27$ & 0.006 \\
OGT (min) & $179 \pm 49$ & $276 \pm 124$ & $<0.001$ \\
IVT rate (\%) & $3,356(89)$ & $1,999(87)$ & 0.462 \\
Recanalization rate (\%) & $1,574(79)$ & $1,774(79)$ & 0.705 \\
\hline
\end{tabular}

Values are presented as mean \pm standard deviation or number (\%).

NIHSS, National Institutes of Health Stroke Scale; ONT, onset-to-needle time; OGT, onset-to-groin time; IVT, intravenous thrombolysis.

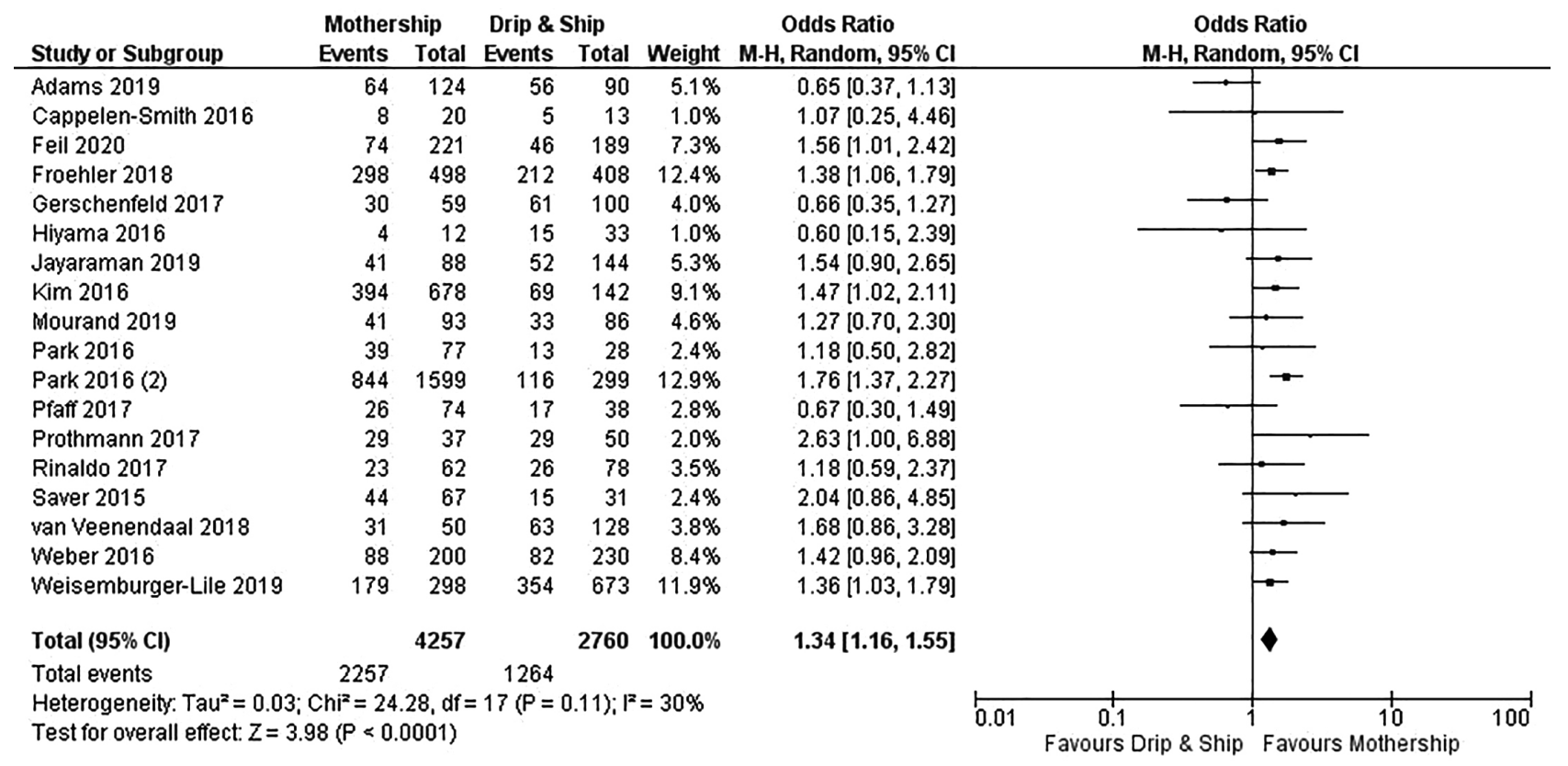

Figure 2. Forest plot showing the association of organizational paradigms with functional independence (modified Rankin Scale 0-2) at 3 months. M-H, Mantel-Haenszel; $\mathrm{Cl}$, confidence interval.

the two organizational paradigms for sex, age, and NIHSS score at admission. On the contrary, DS patients had longer onset-to-treatment timing (Table 2).

Considering the primary endpoint of functional independence at 90 days, MS paradigm was superior to DS model (OR, $1.34 ; 95 \% \mathrm{Cl}, 1.16$ to $1.55 ; I^{2}=30 \%$ ) (Figure 2). More specifically, the rates of functional independence were $53 \%$ and $47 \%$ in the MS and DS organizational paradigms respectively, with a NNT of 29 in favor of MS model (Figure 2). Results from DerSimonian \& Laird model were also confirmed applying the Hartung-Knapp-Sidik-Jonkman model (OR, 1.38; 95\% Cl, 1.20 to 1.59; $\left.P_{\text {neterogeneity }}=0.11\right)$. In the subgroup analysis restricted to studies exploring bridging therapy, the MS model provided marginally higher rate of functional independence $(O R, 1.26$; $95 \% \mathrm{Cl}, 0.98$ to $1.63 ; I^{2}=34 \%$ ) (Figure 3 ).

Meta-regression analysis revealed a significant association between ONT and good functional outcome, with longer ONT being detrimental for recovery (Supplementary Figure 2). Differences of age, clinical severity at presentation, and OGT between study groups did not have significant correlation with functional outcome (Supplementary Figures 3-5).

MS and DS had similar rates of mortality at 90 days (OR, $0.94 ; 95 \% \mathrm{Cl}, 0.78$ to $1.13 ; \mathrm{l}^{2}=28 \%$ ) and successful recanalization $\left(\mathrm{OR}, 0.81 ; 95 \% \mathrm{Cl}, 0.54\right.$ to $1.21 ; \mathrm{I}^{2}=80 \%$ ) (Supplementary 


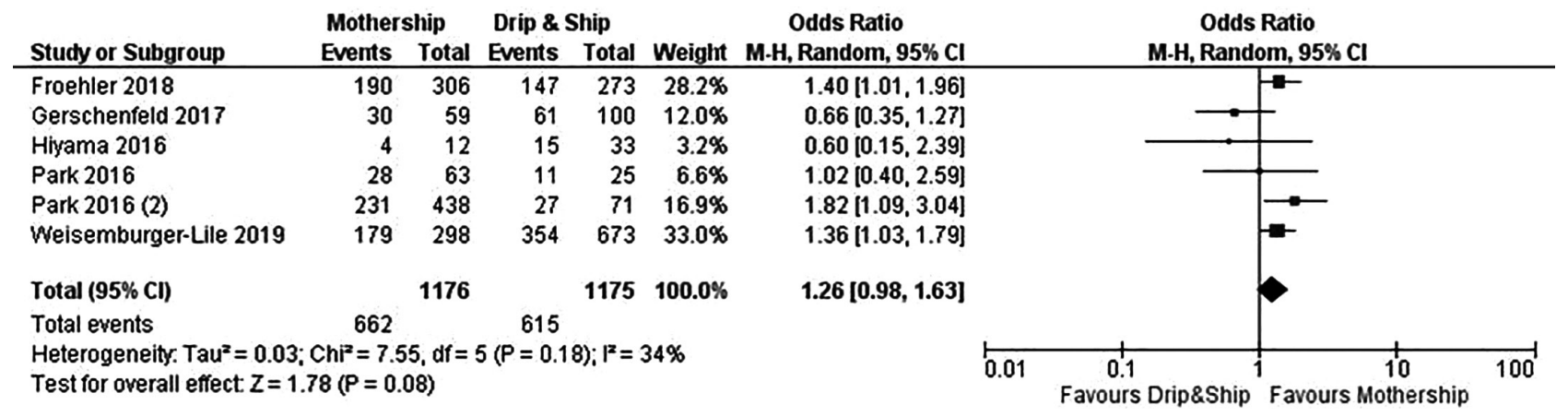

Figure 3. Forest plot showing the association of organizational paradigms with functional independence (modified Rankin Scale 0-2) at 3 months in subgroup of patients treated with bridging therapy (combination of intravenous thrombolysis and endovascular thrombectomy). $\mathrm{M}-\mathrm{H}, \mathrm{Mantel}-\mathrm{Haenszel} ; \mathrm{Cl}$, confidence interval.

Figures 6 and 7). Sensitivity analysis through leave-one out paradigm confirmed results (Supplementary Table 1). Pooling data from all observational studies, excluding RCT, MS was still superior to DS paradigm $1.33(95 \% \mathrm{Cl}, 1.16$ to 1.53$)$ (Supplementary Table 1). Combinatorial analysis further confirmed clustering of possible pooled estimates in favor of MS for primary outcome (Supplementary Figure 8).

Overall, no significant difference was noted in terms of sICH depending on organizational paradigm, with a $6.3 \%$ and $6.7 \%$ in MS and DS models respectively $(\mathrm{OR}, 0.87 ; 95 \% \mathrm{Cl}, 0.70$ to $1.10 ; I^{2}=15 \%$ ) (Supplementary Figure 9). There was substantial heterogeneity for the endpoint of successful recanalization across the included studies ( $P$ for Cochran $0<0.00001$, $\left.1^{2}=80 \%\right)$. There was little or no heterogeneity regarding all the other endpoints.

\section{Discussion}

In this systematic review and meta-analysis including patients with acute ischemic stroke eligible for reperfusion strategies, MS organizational paradigm was associated with a higher rates of functional independence at 3 months compared to DS. Our findings add and put into context previous studies and systematic reviews, ${ }_{1}^{5,628}$ particularly regarding the role of treatment timing on plausible benefit. MS provided better 90-day outcome compared to DS in a previous meta-analysis, which however was limited to eight studies $(n=2,068)$ and provided no meta-regression for timing of treatment. ${ }^{5}$ MS superiority failed to be replicated in a meta-analysis integrating 9 studies $(n=4,127)$, which showed similar performance of the two paradigms. ${ }^{6}$ Reviews put into question MS superiority, ${ }^{28}$ and previous conditional modeling providing suggestions for network organization was consistently limited by assumptions on eligibility, door-to-needle time at PSCs, and standardized rates of recanalization., ${ }^{7,29}$ Our results, deriving from 18 studies ( $n>7,000)$, provide substantial insights in the overall net benefit of MS compared to DS, and detail effects on both good functional outcome, sICH and mortality. Specific, patients treated under MS paradigm had approximately $40 \%$ higher odds of being functionally independent at 3 months, with no increase in the risk of $\mathrm{sICH}$, or mortality. The benefit of MS on functional outcome was further confirmed across sensitivity analysis. Therefore, MS might be preferred to DS whenever local facilities allow the application of this organizational paradigm.

A marginal and non-significant benefit of MS over DS was also found taking into account studies investigating bridging. This finding needs to be looked at with caution. First, the analysis was consistently limited by the sample size and heterogeneity, with large-sample studies clustering towards MS benefit versus small-sample ones distributing in the opposite direction. Second, the fact that such trend emerges together with the lack of relationship between OGT and functional outcome might suggest that what happens before EVT translates on outcome. To this extent, ONT was correlated with functional outcome, and was significantly shorter in MS group, suggesting that the rapid access to reperfusion strategies still represents the principal target to achieve good outcome. Results from this meta-analysis highlight that longer ONT negatively impacted 3-month functional outcome, suggesting that logistics should be appropriately addressed when configuring a stroke network. The fact that age and clinical severity did not impact on the benefit of paradigm choice on functional outcomes argues in favor of common pathways for all stroke patients, independently from age and clinical severity at stroke onset.

Regarding recanalization, a marginal, although not significant, increase in rates of recanalization with DS was found 
compared to MS with substantial heterogeneity detected across included studies. These findings support similar efficacy of the two organizational paradigms, although it might also prompt speculations on possible higher rates of futile recanalization in patients managed via DS, which might be attributable to long transportation-time between primary and comprehensive centers, treatment in late time-windows, and lack of eligibility rechecking at arrival.

Limitations to this meta-analysis might be related to the design of included studies, as well as in the use of tabular data, which precluded further adjustment for confounding factors. Second, our results might not be fully representative of stroke care in the near future, when expansion of tissue-based windows for treatment might consistently refine the advantages of one paradigm over the other. To this extent, implementation of simulation modeling might represent a useful resource to orientate logistics, with appropriate continuous audit/feedback. ${ }^{7}$ Third, meta-regression analysis was performed with the caveat of low number of studies, and therefore results regarding ONT timing should be considered with caution. Finally, meta-analysis did not include geographical factors and different centers performance, which might indeed represent critical details to organize the stroke network. However, given MS associates with improved functional outcome, policies might be implemented to support such paradigm in areas with transportation time to CSC below 45 minutes. The ongoing Transfer to the Local Stroke Center versus Direct Transfer to Endovascular Center of Acute Stroke Patients with Suspected Large Vessel Occlusion in the Catalan Territory (RACECAT; NCT02795962) and PREhospital Routage of Acute STroke Patients With Suspected Large Vessel Occlusion: Mothership Versus Drip and Ship (PRESTO-F; NCT04121013) trials will provide more answers to the open questions comparing MS and DS.

\section{Conclusions}

Patients with acute ischemic stroke eligible for reperfusion strategies might benefit from MS paradigm compared to DS. RCTs are needed to further refine best management taking into account logistics, facilities and resources.

\section{Supplementary materials}

Supplementary materials related to this article can be found online at https://doi.org/10.5853/jos.2020.01767.

\section{Disclosure}

The authors have no financial conflicts of interest.

\section{References}

1. Goyal M, Menon BK, van Zwam WH, Dippel DW, Mitchell PJ, Demchuk AM, et al. Endovascular thrombectomy after largevessel ischaemic stroke: a meta-analysis of individual patient data from five randomised trials. Lancet 2016;387:17231731.

2. Vidale $\mathrm{S}$, Agostoni E. Endovascular treatment of ischemic stroke: an updated meta-analysis of efficacy and safety. Vasc Endovascular Surg 2017;51:215-219.

3. Vidale $\mathrm{S}$, Romoli $\mathrm{M}$, Consoli $\mathrm{D}$, Agostoni EC. Bridging versus direct mechanical thrombectomy in acute ischemic stroke: a subgroup pooled meta-analysis for time of intervention, eligibility, and study design. Cerebrovasc Dis 2020;49:223-232.

4. Powers WJ, Rabinstein AA, Ackerson T, Adeoye OM, Bambakidis NC, Becker K, et al. 2018 Guidelines for the early management of patients with acute ischemic stroke: a guideline for healthcare professionals from the American Heart Association/American Stroke Association. Stroke 2018; 49:e46-e110.

5. Ismail M, Armoiry X, Tau N, Zhu F, Sadeh-Gonik U, Piotin M, et al. Mothership versus drip and ship for thrombectomy in patients who had an acute stroke: a systematic review and meta-analysis. J Neurointerv Surg 2019;11:14-19.

6. Ciccone $A$, Berge $E_{1}$ Fischer U. Systematic review of organizational models for intra-arterial treatment of acute ischemic stroke. Int J Stroke 2019;14:12-22.

7. Holodinsky JK, Williamson TS, Demchuk AM, Zhao H, Zhu L, Francis $\mathrm{MJ}$, et al. Modeling stroke patient transport for all patients with suspected large-vessel occlusion. JAMA Neurol 2018;75:1477-1486.

8. Moher D, Liberati A, Tetzlaff J, Altman DG; PRISMA Group. Preferred reporting items for systematic reviews and metaanalyses: the PRISMA statement. PLoS Med 2009;6:e1000097.

9. Viechtbauer W. Conducting meta-analyses in $\mathrm{R}$ with the metafor package. J Stat Softw 2010;36:1-48.

10. Adams KM, Burns PA, Hunter A, Rennie I, Flynn PA, Smyth G, et al. Outcomes after thrombectomy in belfast: mothership and drip-and-ship in the real world. Cerebrovasc Dis 2019; 47:231-237.

11. Feil K, Rémi J, Küpper C, Herzberg M, Dorn F, Kunz WG, et al. Drip and ship for mechanical thrombectomy within the Neurovascular Network of Southwest Bavaria. Neurology 2020;94:e453-e463. 
12. Jayaraman $M V$, Hemendinger $M L$, Baird GL, Yaghi S, Cutting $\mathrm{S}$, Saad $A$, et al. Field triage for endovascular stroke therapy: a population-based comparison. J Neurointerv Surg 2020;12: 233-239.

13. Weisenburger-Lile $D$, Blanc $R$, Kyheng $M$, Desilles JP, Labreuche J, Piotin $M$, et al. Direct admission versus secondary transfer for acute stroke patients treated with intravenous thrombolysis and thrombectomy: insights from the endovascular treatment in ischemic stroke registry. Cerebrovasc Dis 2019;47:112-120.

14. Mourand I, Malissart P, Dargazanli C, Nogue E, Bouly S, Gaillard $\mathrm{N}$, et al. A regional network organization for thrombectomy for acute ischemic stroke in the anterior circulation; timing, safety, and effectiveness. J Stroke Cerebrovasc Dis 2019;28:259-266.

15. Saver $\mathrm{J}$, Goyal $M$, Bonafe $A$, Diener HC, Levy El, Pereira VM, et al. Stent-retriever thrombectomy after intravenous t-PA vs. t-PA alone in stroke. N Engl J Med 2015;372:2285-2295.

16. Pfaff J, Pham $M$, Herweh $C$, Wolf $M$, Ringleb PA, Schönenberger $S$, et al. Clinical outcome after mechanical thrombectomy in non-elderly patients with acute ischemic stroke in the anterior circulation: primary admission versus patients referred from remote hospitals. Clin Neuroradiol 2017;27: 185-192.

17. Froehler MT, Saver JL, Zaidat 00, Jahan R, Aziz-Sultan MA, Klucznik RP, et al. Interhospital transfer before thrombectomy is associated with delayed treatment and worse outcome in the STRATIS registry (systematic evaluation of patients treated with neurothrombectomy devices for acute ischemic stroke). Circulation 2017;136:2311-2321.

18. Kim DH, Bae HJ, Han MK, Kim BJ, Park SS, Park TH, et al. Direct admission to stroke centers reduces treatment delay and improves clinical outcome after intravenous thrombolysis. J Clin Neurosci 2016;27:74-79.

19. Cappelen-Smith $C_{1}$ Cordato $D$, Calic $Z$, Cheung $A$, Wenderoth J. Endovascular thrombectomy for acute ischaemic stroke: a real-world experience. Intern Med J 2016;46:1038-1043.

20. Weber R, Reimann G, Weimar C, Winkler A, Berger K, Nord-

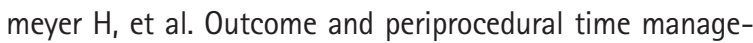

ment in referred versus directly admitted stroke patients treated with thrombectomy. Ther Adv Neurol Disord 2016;9: 79-84.

21. Gerschenfeld G, Muresan IP, Blanc $R$, Obadia $M$, Abrivard $M$, Piotin $M_{1}$ et al. Two paradigms for endovascular thrombectomy after intravenous thrombolysis for acute ischemic stroke. JAMA Neurol 2017;74:549-556.

22. Hiyama N, Yoshimura S, Shirakawa M, Uchida K, Oki Y, Shindo $S$, et al. Safety and effectiveness of drip, ship, and retrieve paradigm for acute ischemic stroke: a single center experience. Neurol Med Chir (Tokyo) 2016;56:731-736.

23. Park MS, Yoon W, Kim JT, Choi KH, Kang SH, Kim BC, et al. Drip, ship, and on-demand endovascular therapy for acute ischemic stroke. PloS One 2016;11:e0150668.

24. Park MS, Lee JS, Park TH, Cho YJ, Hong KS, Park JM, et al. Characteristics of the drip-and-ship paradigm for patients with acute ischemic stroke in South Korea. J Stroke Cerebrovasc Dis 2016;25:2678-2687.

25. Rinaldo L, Brinjikji W, McCutcheon BA, Bydon M, Cloft $H_{\text {, }}$ Kallmes DF, et al. Hospital transfer associated with increased mortality after endovascular revascularization for acute ischemic stroke. J Neurointerv Surg 2017;9:1166-1172.

26. Prothmann S, Schwaiger BJ, Gersing AS, Reith W, Niederstadt T, Felber A, et al. Acute Recanalization of ThromboEmbolic Ischemic Stroke with pREset (ARTESp): the impact of occlusion time on clinical outcome of directly admitted and transferred patients. J Neurointerv Surg 2017;9:817-822.

27. Van Veenendaal P, Yan B, Churilov L, Dowling R, Bush S, Mitchell P. Endovascular clot retrieval by hub-and-spoke service delivery is feasible compared with direct-to-mothership. Cerebrovasc Dis 2018;46:172-177.

28. Détraz L, Ernst M, Bourcier R. Stroke transfer and its organizational paradigm: review of organizational paradigms and the impact on outcome. Clin Neuroradiol 2018;28:473-480.

29. Holodinsky JK, Williamson TS, Kamal N, Mayank D, Hill MD, Goyal M. Drip and ship versus direct to comprehensive stroke center: conditional probability modeling. Stroke 2017;48: 233-238. 


\section{Supplementary material}

\section{Boolean syntax for literature search in PubMed database}

(((mothership[title/abstract]) OR (MS[title/abstract])) OR ((drip and ship[title/abstract]) OR (DS[title/abstract])) OR (hub and spoke[title/abstract])) AND (stroke[title/abstract] OR (cerebrovascular disease*[title/abstract]))
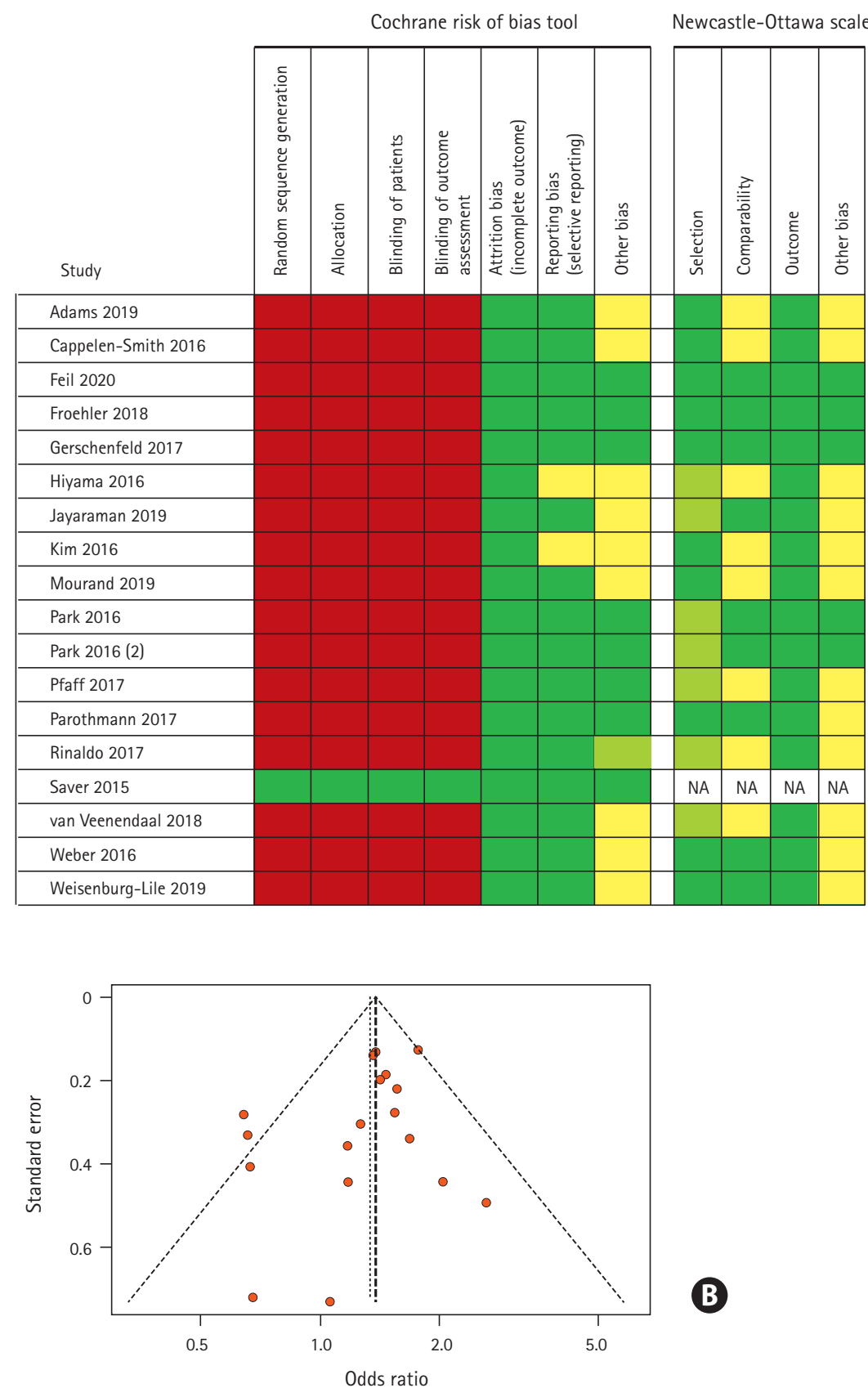

B

Supplementary Figure 1. Risk of bias summary (A) and publication bias Funnel plot (B). Color-based legend: red=high risk of bias, yellow=mild risk of bias, light green=slight risk of bias, dark green=low risk of bias. NA, not available. 


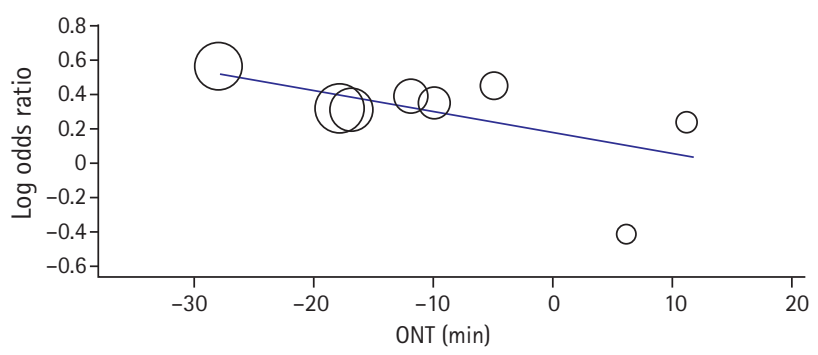

Supplementary Figure 2. Meta-regression analysis for differences of onset-to-needle time (ONT) means between study level groups. Coefficient: -0.013 (95\% confidence interval, -0.025 to -0.00 ); $S E, 0.006 ; P=0.048$.

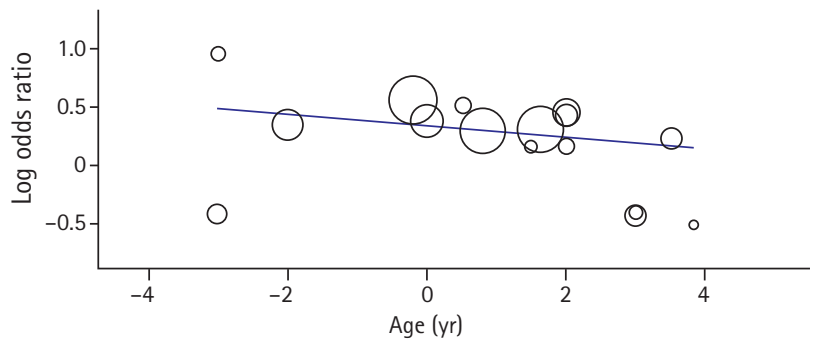

Supplementary Figure 4. Meta-regression analysis for differences of age. Coefficient: -0.051 (95\% confidence interval, -0.125 to 0.002 ); SE, 0.004; $P=0.179$.

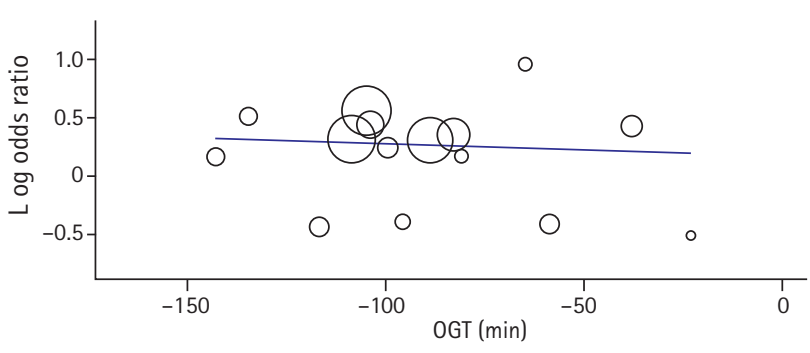

Supplementary Figure 3. Meta-regression analysis for differences of onset-to-groin time (OGT) means between study level groups. Coefficient: -0.001 (95\% confidence interval, -0.007 to 0.005 ); $S E, 0.003 ; P=0.9$.

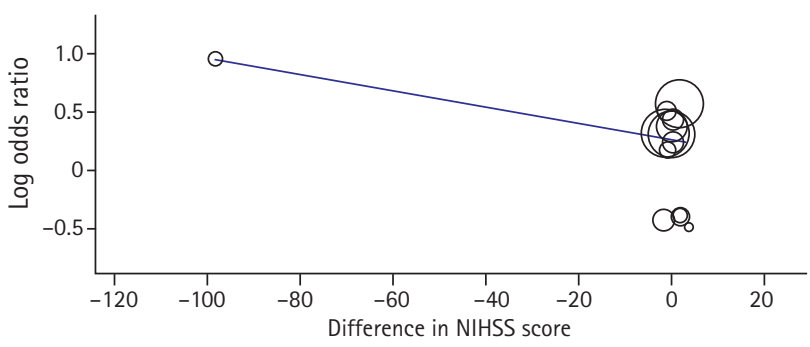

Supplementary Figure 5. Meta-regression analysis for differences of clinical severity. Coefficient: -0.007 ( $95 \%$ confidence interval, -0.001 to 0.003 ); $\mathrm{SE}, 0.005 ; P=0.176$. NIHSS, National Institutes of Health Stroke Scale.

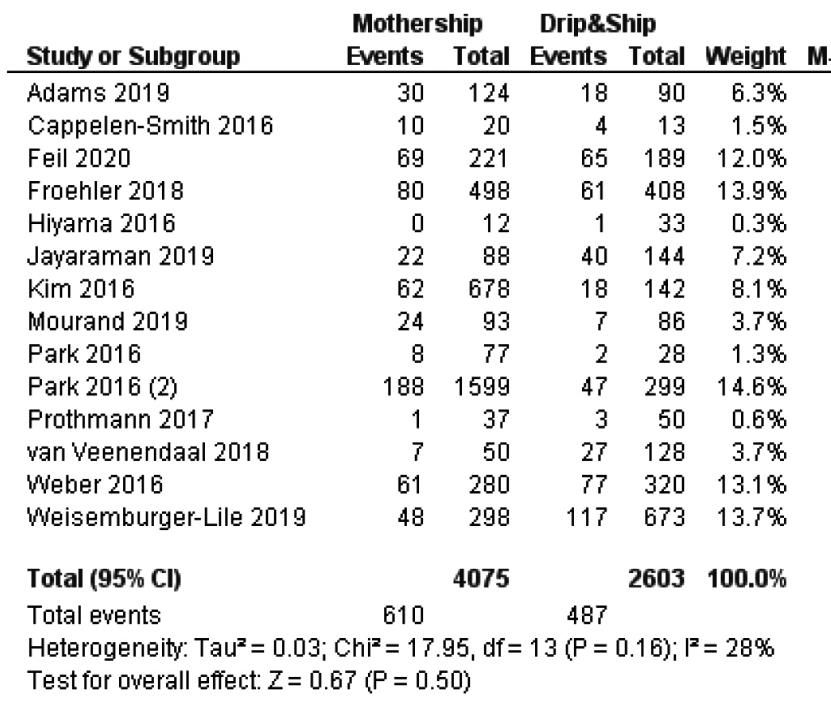

Odds Ratio

, Random, 95\% Cl

$1.28[0.66,2.47]$

$2.25[0.52,9.77]$

$0.87[0.57,1.31]$

$1.09[0.76,1.56]$

$0.87[0.03,22.72]$

$0.87[0.47,1.59]$

$0.69[0.40,1.21]$

$3.93[1.59,9.67]$

$1.51[0.30,7.57]$

$0.71[0.51,1.01]$

$0.44[0.04,4.36]$

$0.61[0.25,1.50]$

$0.88[0.60,1.29]$

$0.91[0.63,1.32]$

$0.94[0.78,1.13]$

Supplementary Figure 6. Forest plot showing association of organizational paradigm with mortality at 3 months. M- $\mathrm{H}$, Mantel-Haenszel; Cl, confidence interval. 


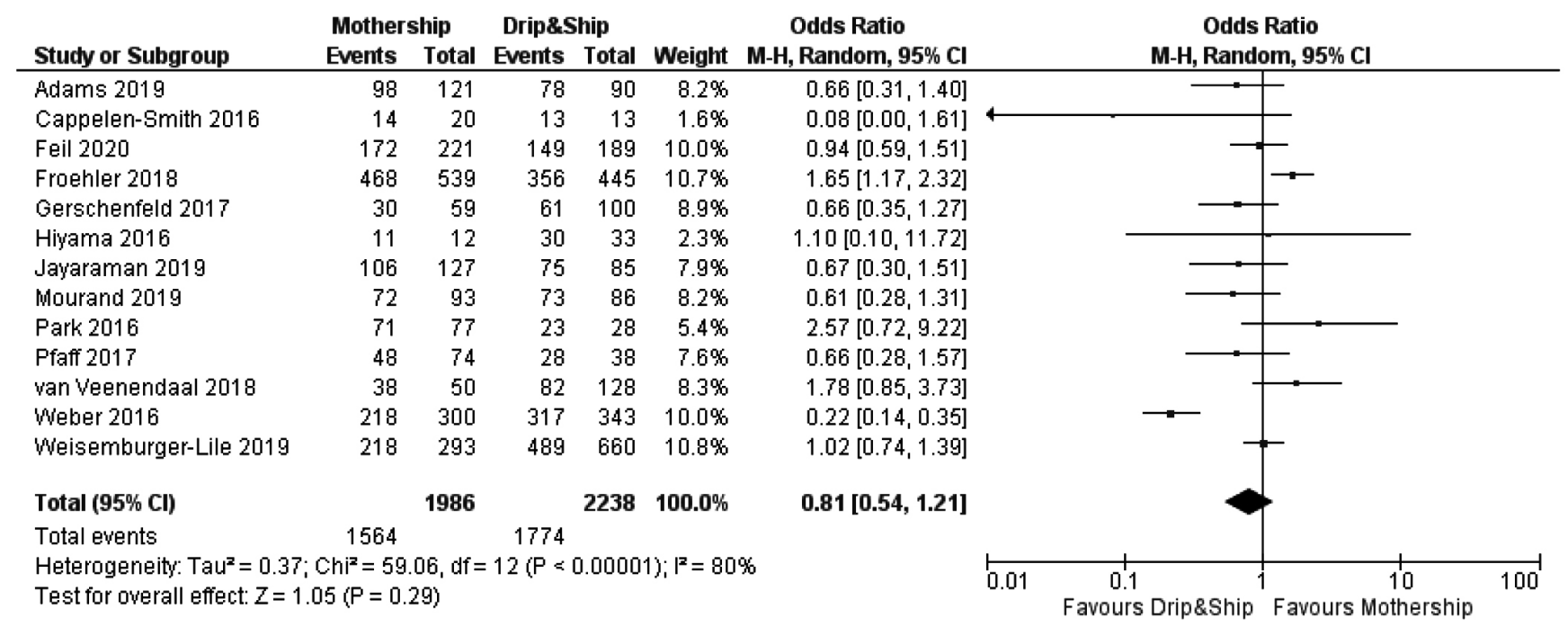

Supplementary Figure 7. Forest plot showing association of organizational paradigm with recanalization. $\mathrm{M}-\mathrm{H}$, Mantel-Haenszel; $\mathrm{Cl}$, confidence interval.

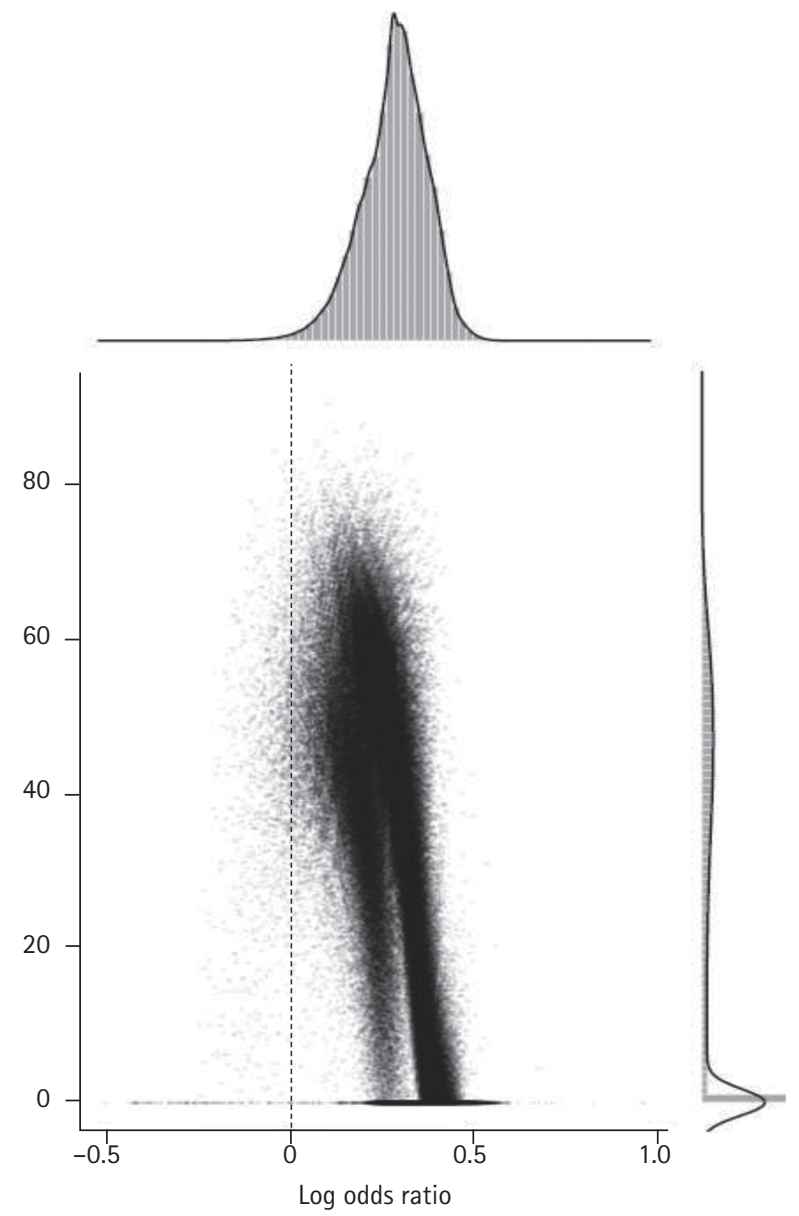

Supplementary Figure 8. Combinatorial analysis showing distribution of effect size and heterogeneity. The distribution of log odds ratio is in consistently in favor of mothership versus drip \& ship paradigm for the primary outcome considered (good functional outcome, modified Rankin Scale 0-2). 


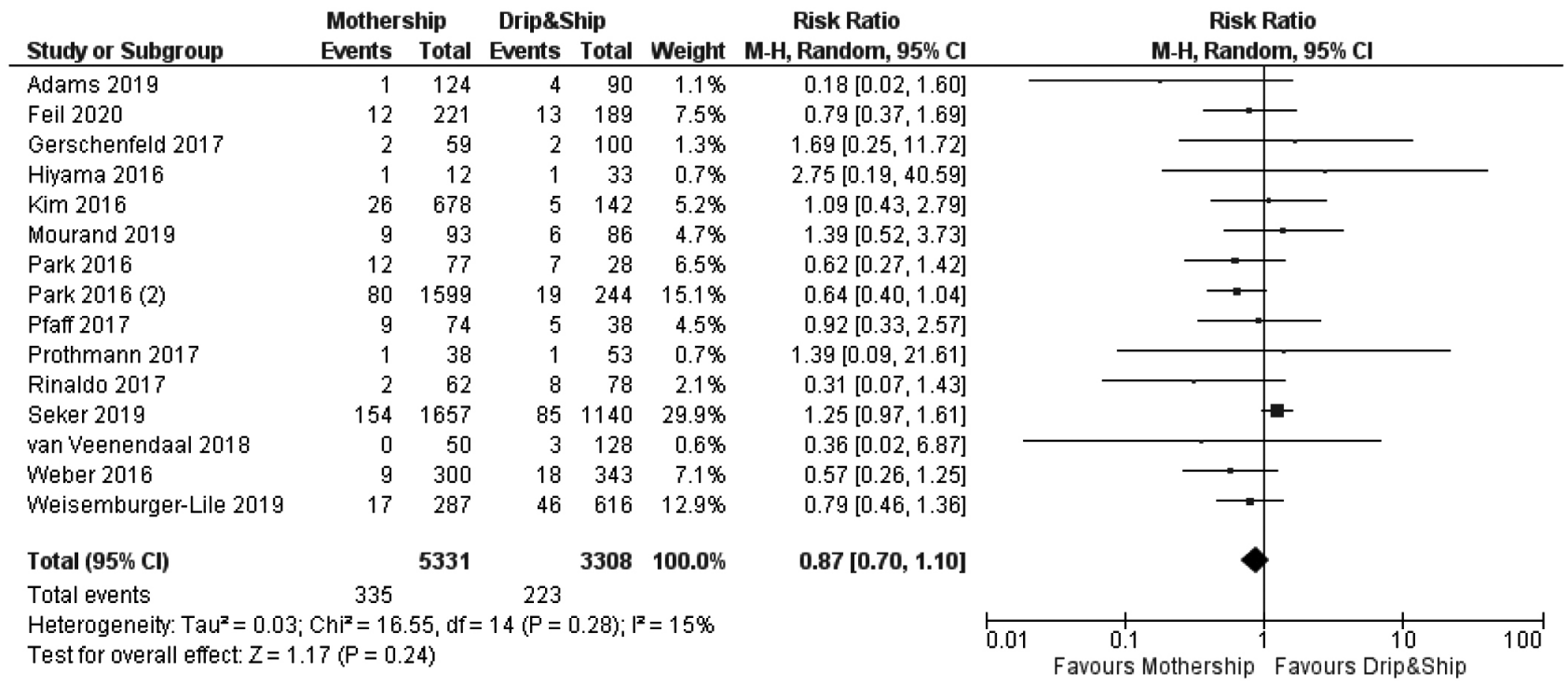

Supplementary Figure 9. Forest plot for symptomatic intracerebral haemorrhage. $\mathrm{M}-\mathrm{H}$, Mantel-Haenszel; $\mathrm{Cl}$, confidence interval.

Supplementary Table 1. Leave-one out sensitivity analysis

\begin{tabular}{|c|c|c|c|}
\hline Study & OR $(95 \% \mathrm{Cl})$ & $\operatorname{tau}^{2}$ & $\mathrm{I}^{2}$ \\
\hline Adams et al. $(2019)^{10}$ & $1.43(1.28-1.59)$ & 0.000 & 0.00 \\
\hline Cappelen-Smith et al. (2016) & $1.35(1.18-1.55)$ & 0.018 & 24.82 \\
\hline Feil et al. (2020) $)^{11}$ & $1.32(1.14-1.54)$ & 0.026 & 30.08 \\
\hline Froehler et al. (2017) ${ }^{17}$ & $1.33(1.13-1.56)$ & 0.032 & 32.67 \\
\hline Gerschenfeld et al. (2017) ${ }^{21}$ & $1.4(1.24-1.58)$ & 0.005 & 8.39 \\
\hline Hiyama et al. $(2016)^{22}$ & $1.36(1.19-1.55)$ & 0.016 & 22.64 \\
\hline Jayaraman et al. (2020) ${ }^{12}$ & $1.33(1.15-1.54)$ & 0.024 & 29.52 \\
\hline Kim et al. (2016) ${ }^{18}$ & $1.32(1.13-1.54)$ & 0.028 & 31.67 \\
\hline Mourand et al. (2019) $)^{14}$ & $1.35(1.17-1.55)$ & 0.022 & 27.91 \\
\hline Park et al. (2016) $)^{23}$ & $1.35(1.17-1.55)$ & 0.020 & 25.87 \\
\hline Park et al. (2016) $)^{24}$ & $1.31(1.16-1.48)$ & 0.000 & 0.00 \\
\hline Pfaff et al. (2017) ${ }^{16}$ & $1.38(1.22-1.57)$ & 0.011 & 16.24 \\
\hline Prothmann et al. $(2017)^{26}$ & $1.33(1.16-1.53)$ & 0.019 & 25.70 \\
\hline Rinaldo et al. $(2017)^{25}$ & $1.35(1.17-1.55)$ & 0.020 & 26.31 \\
\hline Saver et al. $(2015)^{15}$ & $1.33(1.16-1.53)$ & 0.021 & 26.75 \\
\hline van Veenendaal et al. $(2018)^{27}$ & $1.33(1.15-1.53)$ & 0.022 & 28.14 \\
\hline Weber et al. $(2016)^{20}$ & $1.33(1.14-1.55)$ & 0.028 & 31.72 \\
\hline Weisenburger-Lile et al. (2019) ${ }^{13}$ & $1.33(1.13-1.56)$ & 0.032 & 32.62 \\
\hline
\end{tabular}

